\title{
Interaction Research on the Smart Power Consumption
}

\author{
Suxiang Zhang ${ }^{1}$, Jianming Liu ${ }^{1}$, Suxian Zhang ${ }^{2}$, Jinping Cao ${ }^{1}$ \\ ${ }^{1}$ State Grid Information \& Telecommunication Co., Ltd. Beijing \\ ${ }^{2}$ Department of foreign language of Hebei university, Baoding, China \\ Email: zsuxiang@163.com
}

Received March, 2013

\begin{abstract}
Due to the rapid growth of the air conditioning load of the residents had bigger impact on power grid safety and power smooth operation, in order to improve energy conservation and reduce emissions of the residents, many countries had studied the home energy management system (HEMS) in the world. In this paper, based on the summary of the work of other countries, the HEMS research and practice situation of China were discussed, combined with the national development and reform commission (NDRC) project, the power consumption visualization empirical research had been carried on in different cities in 2012, the interaction results show that the load can be reduced by an average of 6.67\%, which proved that the electricity interactive was very interesting for long-term strategy of energy conservation and emissions reduction.
\end{abstract}

Keywords: HEMS; Smart Power Consumption; Load Regulation and Control; Power Consumption Visualization

\section{Introduction}

A smart grid is an electrical grid that uses information and communications technology to gather and act on information, such as information about the behaviors of suppliers and consumers, in an automated fashion to improve the efficiency, reliability, economics, and sustainability of the production and distribution of electricity [1]. The smart grid also allows for systematic communication between suppliers and consumers, and permits both to be more flexible and sophisticated in their operational strategies. The overall effect is a signal that awards energy efficiency and energy consumption that is sensitive to the time-varying limitations of the supply.

In recent years, with the rapid development of economy and the improvement of people's living standard, power tense situation had been more and more obvious, Load management [2] is very essential to the operation of power companies, which can enhance the energy-efficient and reliable operation of power system, and we need to build the flexible and intelligent energy systems, that are able to meet the goals set for reducing $\mathrm{CO} 2$ emissions as well as to ensure security of supply in the future.

In this paper, we paid some attentions for home energy management systems (HEMS) in the smart grid, the contents of this paper are as following: section 2 introduced some practices in other countries; based on the modern communication and information processing, China HEMS was proposed and discussed in section 3 ; in section 4 , we introduce some achievements in our HEMS work; finally is conclusions.

\section{Related Works in Other Countries}

Controlling electricity consumption is one of the key features within future smart community. HEMS have played a role this by controlling Home appliances through Home Appliance control interface. Many countries in the world have studied HEMS as following:

In South Korean [3], the increasing demand of electricity along with increased efficiency requirement creates new opportunities for the development of demand side management programs. However, due to the low residential electricity costs, consumers lack the understanding of the smart grid, reluctant to buy smart grid related equipment and products, making the smart grid market it are difficult to generate revenues. South Korea's knowledge economy ministry had decided to invest 2547 billion won from 2009 to 2012 in developing smart grid commercial technology, the sensors were installed in power stations, transmission equipment and electrical appliances product, which was known as "green electricity IT" project.

In Canada [4], HEMS are integral to the aspects of Smart Grid that are on the customer side of the electricity meter and will provide the means for customers to exert control over and maximize the efficiency of energy use within their homes. Ontario Power Company had deployed smart meters over 80 distribution companies; 
smart meter installation number has reached 4.6 million. Ontario Power Company had cooperation with Trilliant, Elster, Sensus, SilverSpring and Tantalus company, who had deployed five different types AMI respectively, which can make HEMS market application to became reality, to help residents are more "smart" electricity.

In USA [5] the Green Button initiative is an industry-led effort that responds to a White House call-to-action to provide utility customers with easy and secure access to their energy usage information in a consumerfriendly and computer-friendly format. Customers are able to securely download their own detailed energy usage with a simple click of a literal "Green Button" on electric utilities' websites. With their own data in hand, consumers can take advantage of a growing array of online services to help them manage energy use and save on their bills.

In Japan [6] The Ministry of Economy, Trade and Industry (METI) has decided to recommend Echonet-Lite as the standard interface for connecting electric appliances and electronic equipment in the home to a HEMS. METI had defined the HEMS protocols and commands for connection with appliances equipped with the Echonet-Lite interface, so that home electric appliances with the Echonet-Lite interface can be commercialized in time to contribute to power saving. With increasing awareness of environmental issues and concerns over the supply of electricity in Japan since the Great East Japan Earthquake, energy saving measures for the home is becoming a pressing need. In response to that need, Panasonic has developed the Smart Home Energy Management System (SMARTHEMS) to help create, store, use and manage energy efficiently. Data concerning electricity, gas and water consumption is measured in real time, and users can for example monitor the current charging status of storage batteries and solar power generation systems and see the CO2 balance for the entire home, giving an insight into energy usage and ways of further saving energy in the home [7].

\section{Interaction Research in China}

\subsection{Definition of the HEMS}

In China [8], the Smart grid is the highly integrated by the modern technology and the strong network, which can maximize accept clean energy, achieve a low carbon green life, and improve the stability of the power grid, so it is the new modern grid. In the power consumption link, in this paper, we only had studied on HEMS, firstly, we will give the definition of the HEMS: the network interaction and instant connection can be realized between users and family electrical equipments, and between users and power grid companies through a series of equipment with the following functions: energy consumption acquisition, equipment control, interaction with users, and interaction with the grid, and realize the management of home energy consumption to help the user saving energy.

The HEMS system has played an important role to achieve the family energy efficiency management, which had decided the interactive manner, communication technology and procedure between the users and the power grid, and between intelligent home appliances and the power grid.

HEMS were installed in the residents home as shown in Figure 1, the electricity information using wirelessly (433 MHZ) way were sent to the HEMS Intelligent gateway by using of the smart sockets, which included washing machine, refrigerator, air conditioner and other high-power power consumption equipment, so we completed the data collection tasks. Users can interact through HEMS terminals (using WiFi way) to see their electricity data; Moreover, the real-time power consumption information were remotely transmitted to the master station through HEMS intelligent gate and optical fiber composite low voltage cables (OPLC), when people want to know his power consumption information, they can brows terminal; at the same time, other service can be proposed by other companies using our HEMS system, such as seeing hd movie, order foods, telemedicine and so on. Moreover, clean energy can be used in family, transmission and distribution infrastructure will be better able to handle possible bidirectional energy flows, allowing for distributed generation such as from photovoltaic panels on building roofs.

\subsection{Key Products}

To realize the HEMS, the key equipments were researched such as air-conditioning, air-conditioning fan, these equipments need information interaction with user, for example, the user can know the power consumption of the air-conditioning and load period, but now, lack of

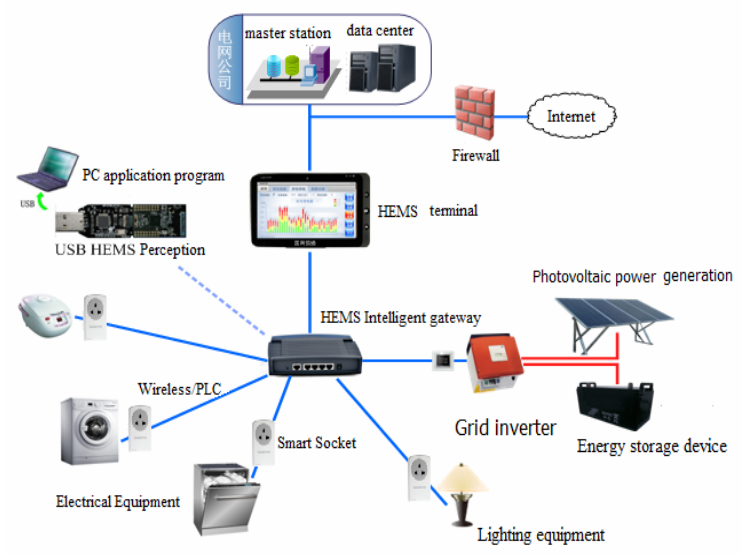

Figure 1. HEMS family architecture. 
the functions in China, the Media manufacture etc. had produced some intelligent home appliance, but the price is very expensive. What can we do more? According to HEMS system application requirements, the HEMS terminal, smart socket, intelligent gateway and other hardware products had been researched. HEMS terminal(as shown in Figure 2) can be used to monitor and manage the family electrical equipment, which can collect and analyze for the electric power quality and power consumption information, to guide the users for the reasonable power consumption, to adjust grid load shift, and realize the smart power consumption between the power grid and users. The intelligent gateway (as shown in Figure 3) is the family control hub, mainly to achieve the information collection and forwarding, such as security alarm, home appliances, electric data and all kinds of control information. It is interacted with the HEMS terminal and other products through the network. Smart socket (as shown in Figure 4) can acquire the real-time electric power data, and remotely control on-off state by using the equipment (HEMS terminal or computer) and can remotely monitor appliances work state to help user reasonable electric consumption. Some energy management software had also been developed as shown in Figure 5.

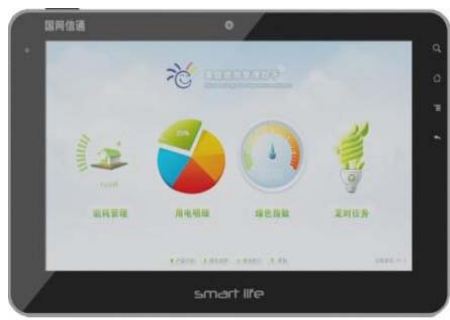

Figure 2. HEMS terminal.

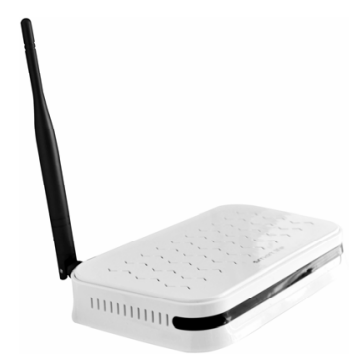

Figure 3. HEMS intelligent gateway.

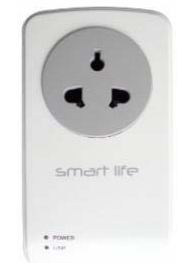

Figure 4. Smart socket.

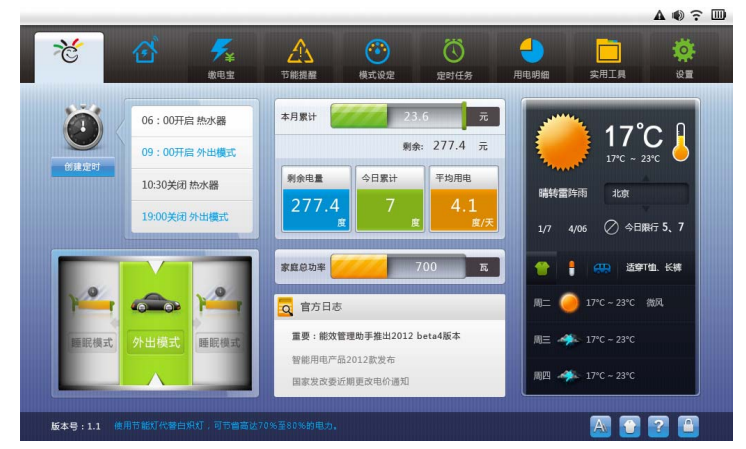

Figure 5. Home energy efficiency management assistant.

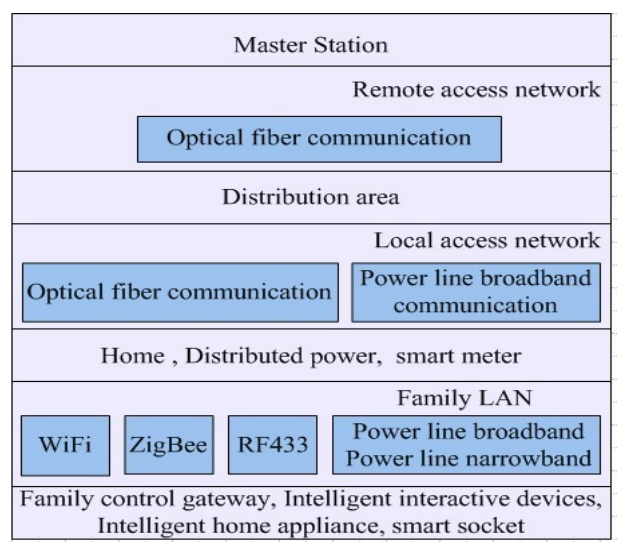

Figure 6. Communication architecture of HEMS.

In Figure 6, we show that the communication architecture of HEMS, In the HEMS, many communication technologies had been used. In the family LAN, such as Wi-Fi, Zigbee, RF433, power line broadband or power line narrowband and so on. The information comes from home distributed power and the smart meter will be integrated in the local access network, here, optical communication and the power line broadband can be used. Through the distribution area, here, using of the optical fiber communication. The final information can be remotely transferred to the master station.

\subsection{Communication Architecture of HEMS}

To reduce demand during the high cost peak usage periods, communications and metering technologies inform smart devices in the home when energy demand is high and track how much electricity is used and when it is used. It also gives utility companies the ability to reduce consumption by communicating to devices directly in order to prevent system overloads.

In the HEMS, many communication technologies had been used. In the family LAN, such as Wi-Fi, Zigbee, RF433, power line broadband or power line narrowband and so on. The information comes from home distributed power and the smart meter will be integrated in the local 
access network, here, optical communication and the power line broadband can be used. Through the distribution area, here, using of the optical fiber communication. The final information can be remotely transferred to the master station.

\section{Further Work}

In July 2011, the national development and reform commission suggests because the air conditioning load of the residents and business users rapid crease, cause to the great influence of the power grid security and stable operation, how to control the air conditioning load power is the research content of the power demand side management. To research this, in 2012, we had chosen the four cities, Beijing, Shanghai, Nanchang and Yinchuan and recruited some volunteers. In these cities, smart communities had been built and many people had installed HEMS in their home, we carried on the empirical research for power consumption visualization to achieve the influence of power consumption visualization on the user behavior and the electric power load; when the power load is peak, the company will send a message to these volunteers, the volunteers would like to close the air conditioning and other high power appliances in time, our experiment time is in 2012.6-2012.9, the Load reduction results were shown in Table 1(not include Nanchang).

From the different cities, we knew that the load reduction rate had been realized with the different levels, which showed the power consumption visualization could enhance the user saving energy sense, and further reduce the difference between peak and valley and Smooth load curve.

Table 1. Empirical research results.

\begin{tabular}{clc}
\hline \multicolumn{1}{c}{ City } & \multicolumn{1}{c}{ Data term } & Kw \\
\hline \multirow{2}{*}{ Beijing } & The Maximum load & 37.46 \\
& The minimum load & 4.83 \\
& The difference between Peak and valley & 32.63 \\
& Load reduction rate & $6.82 \%$ \\
& The Maximum load & 44.9 \\
Shanghai & The minimum load & 5.49 \\
& The difference between Peak and valley & 39.41 \\
& Load reduction rate & $8.77 \%$ \\
& The Maximum load & 25.7 \\
& The minimum load & 1.64 \\
& The difference between Peak and valley & 24.06 \\
& Load reduction rate & $4.43 \%$ \\
\hline
\end{tabular}

\section{Conclusions}

The interaction on the smart power consumption can be researched; we gave the definition of home energy management system in this paper, discussed its related HEMS system structure and communication technology, and elaborated the practice and further work in the field of HEMS. Through an empirical study for power consumption visualization in the different cities, experiment showed that interaction could reduce electricity load difference between peak and valley, and smooth the load curve. Along with further construction and promotion of the smart grid, the energy consciousness of the user can be improved, and the slogan "intelligent power consumption, better life" will be became the main goal of the life.

\section{Acknowledgements}

This work was supported by National High-tech R\&D Program of China (863 Program) (No. 2011AA05A116), National major projects of science and technology: Collaborative network control platform and the key technology of ubiquitous networks under multi-terminal (2011ZX03005-004-01); the National Basic Research Program of China (973 Program): Basic theory and practice research of Internet of Things (2011CB302900).

The work was also supported by science and technology project of State Grid Corporation (Smart power consumption empirical study)

\section{REFERENCES}

[1] U.S. Department of Energy, "Smart Grid / Department of Energy,"

http://energy.gov/oe/technology-development/smart-grid. Retrieved 2012-06-18.

[2] A. K. Topalli , I. Erkmen and I. Topalli, "Intelligent Short Term Load Forecasting in Turkey,” International Journal of Electrical Power \& Energy Systems, Vol. 28, No. 7, 2006, pp. 437-447. doi:10.1016/j.ijepes.2006.02.004

[3] H. Y. Lin, J. Zhang, K. P. Xu and X. J. Pi, "Design of Interactive Service Platform for Smart Power Consumption,” Power System Technology, Vol. 36, No. 7, 2012, pp. 255-259.

[4] John O’Neill, "PASC HEMS Workshop Smart Grid and HEMS in Canada," The First Workshop on HEMS Within Smart Grid, 2012, 6, South Korean.

[5] A. jillavenkates, "Home Energy Management Systems in The Smart Grid Workshop," The First Workshop on HEMS within Smart Grid, 2012, 6, South Korean.

[6] Y. Shobatake, "ECHONET as a Basis of HEMS System," The First Workshop on HEMS Within Smart Grid, 2012, 6, South Korean.

[7] http://news.panasonic.net/archives/2012/0912_13250.htm

[8] S. X. Zhang, "The Research and Practice of HEMS in China," The First Workshop on HEMS Within Smart Grid, 2012, 6, South Korean. 